\title{
Association of Financial Worry and Material Financial Risk with Short-Term Ambulatory Healthcare Utilization in a Sample of Subsidized Exchange Patients
}

\author{
Salene M. W. Jones, PhD, MA' , Matthew P. Banegas, PhD, MPH, MS ${ }^{2}$, \\ John F. Steiner, MD, MPH ${ }^{3}$, Emilia H. De Marchis, MD, MAS 4 , Laura M. Gottlieb, MD, MPH', \\ and Adam L. Sharp, MD, MSc ${ }^{5,6}$
}

\begin{abstract}
${ }^{1}$ Fred Hutchinson Cancer Research Center, Seattle, WA, USA; ${ }^{2}$ Kaiser Permanente Oregon Center for Health Research, Portland, OR, USA; ${ }^{3}$ Kaiser Permanente Colorado Institute for Health Research, Aurora, CO, USA; ${ }^{4}$ Department of Family and Community Medicine, University of California San Francisco, San Francisco, CA, USA; ${ }^{5}$ Research and Evaluation Department, Kaiser Permanente Southern California, Pasadena, CA, USA; ${ }^{6}$ Health Systems Science Department, Kaiser Permanente School of Medicine, Pasadena, CA, USA.
\end{abstract}

BACKGROUND: Financial burden can affect healthcare utilization. Few studies have assessed the short-term associations between material (debt, trouble paying rent) and psychological (worry or distress about affording future healthcare) financial risks, and subsequent outpatient and emergency healthcare use. Worry was defined as concerns about affording future healthcare.

OBJECTIVE: Examine whether worry about affording healthcare is associated with healthcare utilization when controlling for material risk and general anxiety

DESIGN: Longitudinal observational study

PARTICIPANTS: Kaiser Permanente members with exchange-based federally subsidized health insurance ( $n$ $=450,45 \%$ response rate)

MAIN MEASURES: Survey measures of financial risks (material difficulty paying for medical care and worry about affording healthcare) and general anxiety. Healthcare use (primary care, urgent care, emergency department, and outpatient specialty visits) in the 6 months following survey completion.

KEY RESULTS: Emergency department and primary care visits were not associated with material risk, worry about affording care, or general anxiety in individual and pooled analyses (all 95\% confidence intervals (CI) for relative risk (RR) included 1). Although no individual predictor was associated with urgent care use (all 95\% CIs for RR included 1), worry about affording prescriptions (relative risk $(\mathrm{RR})=2.01 ; 95 \%$ CI $1.14,3.55)$ and general anxiety $(\mathrm{RR}=0.38 ; 95 \%$ CI $0.15,0.95)$ were significant when included in the same model, suggesting the two confounded each other. Worry about affording healthcare services was associated with fewer specialty care visits $(\mathrm{RR}=0.40 ; 95 \% \mathrm{CI} 0.25,0.64)$ even when controlling for material risk and general anxiety, although general anxiety was also associated with more specialty care visits $(\mathrm{RR}=1.98 ; 95 \% \mathrm{CI}$, $1.23,3.18)$.

Prior Presentations: None

Received May 21, 2020

Accepted December 15, 2020

Published online January 19, 2021
CONCLUSIONS: Screening for both general anxiety and financial worry may assist with specialty care utilization. Identifying these concerns may provide more opportunities to assist patients. Future research should examine interventions to reduce worry about cost of care.

KEYWORDS: economic well-being; financial toxicity; financial burden; financial hardship.

J Gen Intern Med 36(6):1561-7

DOI: $10.1007 / \mathrm{s} 11606-020-06479-6$

(c) Society of General Internal Medicine 2021

\section{INTRODUCTION}

Social determinants of health $(\mathrm{SDOH})$ are the contexts in which people live and work that affect their health, ${ }^{1}$ ranging from quality of life to mortality. ${ }^{2-9}$ Financial burden is among the social risk factors that can adversely impact health. Financial burden can be divided into psychological and material domains. ${ }^{10-12}$ Psychological financial risk includes perceptions and emotions related to financial security, such as worry about affording healthcare and distress from past financial burden. Material financial risk includes trouble paying for basic needs (utilities, housing, medical care) and increases in debt or risk of bankruptcy. The few studies that have examined the concurrent associations of material and psychological financial risks, which we designate as "financial burden," among patients suggest that these factors are both associated with foregoing or delaying medical care and with cost-related nonadherence. ${ }^{13-16}$ However, psychological financial risk is not synonymous with material financial risk. One study of cancer survivors showed that worry does not always align with material risk. $^{17}$

Knowing which types of financial burden affect healthcare utilization could help healthcare systems identify patients at risk for delaying medical care or cost-related nonadherence. The association of financial burden with healthcare utilization might vary based on the types of burden and utilization. Some individuals may worry about affording care and thus postpone 
care to save costs and reduce material risk, despite potential adverse effects on their long-term health. For example, worry about affording healthcare might be associated with lower utilization of preventive services such as cancer screening, which can more easily be delayed than emergency care. However, to our knowledge, no study has examined the role of psychological financial risk in healthcare utilization while accounting for material risk.

Worry about affording healthcare should also be differentiated from general worry or anxiety. Psychological studies have shown that worry needs to be specific to the behavior (healthcare costs in this case) to be predictive of other outcomes. ${ }^{18-20}$ General worry and anxiety may be worsened by a patient's health anxiety that could increase healthcare utilization, ${ }^{21}$ the opposite of the hypothesized association of worry about affording healthcare. To our knowledge, no study has examined how general anxiety differs from material and psychological financial risks in predicting healthcare utilization.

To elucidate the relationship of worry about affording healthcare with healthcare utilization (outpatient visits, emergency room visits, urgent care visits), we analyzed survey responses among members in a large integrated healthcare system who received insurance coverage through a subsidized insurance exchange. ${ }^{22,23}$ Our study had two aims. In aim 1, we examined whether worry about affording healthcare was associated with future healthcare use in the near term, specifically for outpatient, emergency, and urgent care visits. Based on the psychopathology literature, we conceptualized worry as future-focused cognitions (thoughts) about being able to pay for healthcare. ${ }^{24}$ For aim 2, we tested whether the association of worry about affording healthcare services and prescriptions with healthcare utilization remained after controlling for material financial risk and general anxiety. Although a majority of our outcomes were related to use of healthcare services, prescription medications were also included because they are high-cost items and because patients may avoid outpatient care anticipating that outpatient visits would lead to additional costly medications.

\section{METHODS}

\section{Participants and Procedures}

Participants were recruited through Kaiser Permanente Southern California (KPSC). To be eligible, potential participants needed to receive health insurance through the subsidized insurance exchanges, which are run by the state of California. To receive subsidized health insurance through the insurance exchange, KP members were required to be US citizens or permanent residents; have income between 100 and $400 \%$ of the federal poverty level; and have no access to affordable employer-sponsored health insurance, based on income. Additional study eligibility criteria were as follows: able to read and speak English or Spanish; age 18 or older at the time of the survey; a residential address in the KPSC service area to ensure access to KPSC services; and continuous enrollment in KPSC for 9 months or more (except for gaps of 90 days or less) prior to the survey to ensure valid measures of healthcare use before the survey. We randomly selected 1008 of the nearly 135,000 eligible subsidized exchange members in KPSC within three strata, age, gender, and language. The 1008 potential participants were sent a mailed invitation to take the survey online or by phone. Administrative data from the electronic health record (EHR) and claims data for out of network claims were linked to participant surveys. The primary purpose of the survey was to psychometrically evaluate two social risk screening tools and determine which tool was most appropriate for the healthcare exchange population, as this group often does not qualify for other government assistance but still experiences financial burden. The study was powered to use kappa statistics to compare agreement for relatively low base-rate social risks (such as housing instability). The analyses reported in this paper are a secondary analysis of the survey. Institutional review board approval was obtained for study procedures and all participants provided informed consent.

\section{Measures}

Predictors. The survey assessed three sets of predictors. First, worry about affording healthcare (psychological financial burden) was assessed using two items from the Worry about Affording Healthcare Scale, which has been shown to be valid and reliable. ${ }^{25,26}$ The two items asked participants to rate worry about affording ("How worried are you RIGHT NOW, if at all, that..."): (1) healthcare services ("You won't be able to afford the health care services you need") and (2) prescription medications ("You won't be able to afford the prescription drugs you need"), both on a 4-point scale (not at all worried; a little worried; somewhat worried; very worried). The entire Worry about Affording Healthcare Scale is available as supplementary material from the validation study. ${ }^{25,26}$ Second, material financial risk was assessed by asking participants whether they experienced any difficulty paying for medical care in the past 3 months. Participants were asked if they had difficulty with a variety of social risk factors, such as food insecurity and housing instability, and an affirmative response to the medical care item was coded as having difficulty paying for medical care. ${ }^{27-30}$ General anxiety was assessed using the Generalized Anxiety Disorder 2 (GAD2), which has participants rate two symptoms of generalized anxiety (feeling nervous, anxious or on edge; not being able to stop or control worry) on a 4-point scale and is a screening tool for problematic anxiety. ${ }^{31}$ Scores on the GAD2 range from 0 to 6 ; scores of three or more indicate a positive screen for GAD.

Outcomes. Information on healthcare utilization was obtained for the 6 months after the participant completed the survey. Utilization measures included number of emergency department (ED) visits, number of urgent care visits, number 
of primary care outpatient visits, and number of specialist outpatient visits. Any utilization at a KPSC facility was captured in the EHR, and claims were included for utilization at out of network facilities, but utilization that was not billed, such as for self-pay, would not have been included.

Covariates. Additional covariates were obtained from the EHR. These included age, sex, race/ethnicity, Neighborhood Deprivation Index (NDI), ${ }^{5}$ whether they received a costsharing reduction subsidy, and preferred language (English vs. Spanish). The NDI uses Census tract information to estimate level of deprivation in the participants' environment. NDI variables include percent unemployed, percent with less than high school or equivalent education, percent of households with income below $\$ 30,000 /$ year, and percent living on public assistance. Each variable is weighted based on a principal components analysis from the original study. Costsharing reduction subsidies help cover a portion of out-ofpocket costs such as copayments. In addition, healthcare utilization for the 12 months before the survey was obtained to provide a baseline covariate. As some participants only had data for the previous 9 months, prior utilization was calculated based on the amount of data available. We were unable to include comorbidity status as a covariate as $82 \%$ of the sample was missing the comorbidity variable calculated from the health records.

\section{Statistical Analysis}

To test aim 1 (association of financial worry with healthcare utilization), we ran a series of Poisson regressions. Each healthcare utilization outcome was treated as a count variable and each was modeled separately. The two healthcare financial worry items were entered as predictors into separate regressions. Answers to each worry question were dichotomized with "not at all worried" and "a little worried" grouped together and "somewhat worried" and "very worried" grouped together. These analyses controlled for the value of the outcome (number of visits) for the 12 months prior to the survey, age, sex, race/ethnicity, NDI, language, and cost-sharing subsidy. Our age groups were 18 to 26 years old, 27 to 44 years old, 45 to 61 years old, and 62 years and older. Twenty-six years old is important as that is the age above which people can no longer be on their parents' insurance. Sixty-two years old is important as that is the earliest age at which people can access Social Security. To test aim 2 (association of financial worry with healthcare utilization when controlling for material risk and general anxiety), difficulty paying for medical care (material financial risk) and a positive screen for GAD were the independent variables for Poisson regression models, with the same set of covariates. We then ran the regressions from aim 1 again but with all four variables of interest in the same model (worry about paying for prescriptions, worry about paying for healthcare services, trouble paying for medical care, and a positive screen for GAD) for each utilization outcome. Poisson models were estimated using generalized estimating equations with robust standard errors, removing the mean-variance relationship requirement for Poisson models and allowing for under- and overdispersion. ${ }^{32}$ The Poisson models had a condition index value of 3.9, indicating problems with multicollinearity were unlikely. ${ }^{33}$

We have included several additional supplementary analyses in the Appendix. First, we examined the bivariate associations between baseline healthcare use (12 months before the survey) and the financial and anxiety variables on the survey. Second, we included analyses that are similar to our main Poisson regressions except an interaction term was added between baseline healthcare utilization and the predictors of interest (material hardship, worry about affording healthcare, general anxiety). Lastly, we included analyses similar to the main Poisson regressions except an interaction term was added between race/ethnicity and the predictors of interest.

\section{RESULTS}

We approached 1008 potential participants, of whom 450 $(45 \%)$ agreed to participate. Participant demographics are reported in Table 1. Most participants spoke and completed the survey in English (84.4\%). A substantially greater proportion of participants reported worry about affording healthcare services $(51.8 \%)$ and prescriptions $(42.2 \%)$ than material difficulty affording care (14.4\%) or general anxiety (18.7\%). As previously reported, non-responders were more likely to speak Spanish, be male, and not have a diagnosis of depression. ${ }^{34}$ Baseline healthcare utilization and neighborhood characteristics did not differ between responders and non-responders. The main reasons for non-response to the survey were not able to reach participant $(357,35 \%)$, refusal $(158,16 \%)$, and ineligible because they were no longer a KP member (26, $3 \%) .{ }^{34}$ The Pearson correlations between the four predictors (material difficulty affording care, worry about affording services, worry about affording prescriptions, general anxiety) were small ( 0.18 to 0.36$)$ except for the correlation between the two worry about affording healthcare items $(0.75$; Appendix Table 1).

\section{Association with Healthcare Use}

The two financial worry, general anxiety and material financial risk measures, were not statistically associated with ED use, urgent care use, or primary care use when analyzed separately (Table 2). Neither worry about affording prescriptions (relative risk $(\mathrm{RR})=0.62 ; 95 \%$ confidence interval $(\mathrm{CI})$ $0.38,1.01)$ nor material financial risk $(\mathrm{RR}=1.18 ; 95 \% \mathrm{CI}$ $0.64,2.19)$ was associated with specialty care outpatient visits. Worry about affording healthcare services was associated with less specialty care utilization $(\mathrm{RR}=0.46 ; 95 \% \mathrm{CI}, 0.28,0.75)$. General anxiety was associated with more specialty care utilization $(\mathrm{RR}=1.82 ; 95 \% \mathrm{CI}, 1.14,2.90)$. 
Table 1 Characteristics of Survey Participants $(n=450)$

\begin{tabular}{|c|c|c|}
\hline Variable & $N$ & $(\%)$ \\
\hline \multicolumn{3}{|l|}{ Age } \\
\hline 18 to 26 years old & 87 & 19.3 \\
\hline 27 to 44 years old & 112 & 24.9 \\
\hline 45 to 61 years old & 91 & 20.2 \\
\hline 62 or more years old & 160 & 35.6 \\
\hline \multicolumn{3}{|l|}{ Sex } \\
\hline Female & 254 & 56.4 \\
\hline Male & 196 & 43.6 \\
\hline \multicolumn{3}{|l|}{ Race/ethnicity } \\
\hline Black, African-American & 14 & 3.1 \\
\hline Hispanic & 118 & 26.2 \\
\hline Missing & 83 & 18.4 \\
\hline Other (Asian American, Native American, & 64 & 14.2 \\
\hline \multicolumn{3}{|l|}{ Pacific Islander, Other) } \\
\hline White & 171 & 38.0 \\
\hline \multicolumn{3}{|l|}{ Language } \\
\hline English & 380 & 84.4 \\
\hline Spanish & 70 & 15.6 \\
\hline \multicolumn{3}{|l|}{$\begin{array}{l}\text { Material financial difficulty affording medical } \\
\text { care }\end{array}$} \\
\hline Missing & 7 & 1.6 \\
\hline No & 378 & 84.0 \\
\hline Yes & 65 & 14.4 \\
\hline \multicolumn{3}{|l|}{ Worried about affording healthcare services } \\
\hline Missing & 15 & 3.3 \\
\hline No & 202 & 44.9 \\
\hline Yes & 233 & 51.8 \\
\hline \multicolumn{3}{|l|}{ Worried about affording prescriptions } \\
\hline Missing & 15 & 3.3 \\
\hline No & 245 & 54.4 \\
\hline Yes & 190 & 42.2 \\
\hline \multicolumn{3}{|l|}{$\begin{array}{l}\text { Positive screen for generalized anxiety } \\
\text { disorder }\end{array}$} \\
\hline Missing & 16 & 3.6 \\
\hline No & 350 & 77.8 \\
\hline Yes & 84 & 18.7 \\
\hline \multicolumn{3}{|l|}{ Cost-sharing subsidy } \\
\hline No & 304 & 67.6 \\
\hline \multirow{2}{*}{ Yes } & 146 & 32.4 \\
\hline & Median & Range \\
\hline \multirow[t]{2}{*}{ Neighborhood Deprivation Index } & -0.12 & $\begin{array}{l}-1.46 \\
3.67\end{array}$ \\
\hline & Median & $\begin{array}{l}\text { Range } \\
\text { (IQR) }\end{array}$ \\
\hline Primary care visits, 12 months before survey & 1.0 & $0,61(0,3)$ \\
\hline Primary care visits, 6 months after survey & 1.0 & $0,28(0,2)$ \\
\hline Specialty care visits, 12 months before survey & 0 & $0,78(0,1)$ \\
\hline Specialty care visits, 6 months after survey & 0 & $0,55(0,1)$ \\
\hline $\begin{array}{l}\text { Emergency department visits, } 12 \text { months } \\
\text { before survey }\end{array}$ & 0 & $0,4(0,0)$ \\
\hline $\begin{array}{l}\text { Emergency department visits, } 6 \text { months after } \\
\text { survey }\end{array}$ & 0 & $0,6(0,0)$ \\
\hline Urgent care visits, 12 months before survey & 0 & $0,12(0,0)$ \\
\hline Urgent care visits, 6 months after survey & 0 & $0,7(0,0)$ \\
\hline
\end{tabular}

For healthcare use, the before-survey period was 12 months and the after-survey period was 6 months

As shown in Table 3, in the models including financial worry while controlling for general anxiety and material risk, financial worry, general anxiety, and material financial risk were not associated with ED utilization or primary care utilization. Greater worry about affording prescriptions ( $R R=$ $2.01 ; 95 \% \mathrm{CI} 1.14,3.55)$ and lower general anxiety $(\mathrm{RR}=$ $0.38 ; 95 \%$ CI $0.15,0.95)$ were both associated with higher urgent care utilization. Similar to the individual models, greater worry about affording healthcare services was associated with lower specialty care utilization $(\mathrm{RR}=0.40 ; 95 \%$ CI 0.25 , 0.64 ) and higher general anxiety was significantly associated with higher specialty care utilization $(\mathrm{RR}=1.98 ; 95 \% \mathrm{CI} 1.23$,
3.18). Worry about affording prescriptions and material financial risk were not associated with specialty care utilization.

Additional analyses examining potential moderators are reported in the Appendix. Healthcare utilization before the survey was unrelated to the financial variables and general anxiety except urgent care use was associated with material financial difficulty ( $\mathrm{RR}=1.25, p=0.04$; Appendix Table 2). Healthcare utilization before the survey did not moderate the associations between subsequent healthcare utilization and the financial variables and general anxiety (Appendix Table 3) except specialty care use and material hardship $(\mathrm{RR}=6.060, p$ $=0.046$ ), emergency room visits with worry about affording services $(\mathrm{RR}=0.151, p=0.002)$ and worry about affording prescriptions $(\mathrm{RR}=0.247, p=0.032)$, and urgent care visits with worry about affording prescriptions $(\mathrm{RR}=0.348, p=$ 0.020 ). Race and ethnicity did not moderate most associations between subsequent healthcare use and financial variables or general anxiety (Appendix Table 4).

\section{DISCUSSION}

This study prospectively examined associations between worry about affording healthcare services and prescriptions, material financial risk, general anxiety, and future healthcare use. In this sample of low-income patients who received subsidized health insurance, worry about affording healthcare was higher than reported in general population samples. ${ }^{16}$ No significant associations were found between the predictors of interest and either ED or primary care utilization. Although worry about affording prescriptions and general anxiety were associated with urgent care utilization when all four predictors were entered into the model, neither was associated with urgent care use when analyzed separately. This was likely due to a suppressor effect, in which one variable increases the statistical significance of another because they are related. ${ }^{35}$ Worry about affording healthcare services was associated with lower specialty care utilization, while general anxiety was associated with higher specialty care utilization. Thus, our hypothesis that worry about affording healthcare would be associated with less care use was supported for specialty care but not for primary care.

Given the continued reliance on specialty care in the modern medical system, the associations between financial worry about affording healthcare services and future specialty care utilization have important implications. Screening for and addressing material financial risk or general anxiety alone might be inadequate to identify worry about affording healthcare. Worry about affording healthcare services may still need to be addressed to improve patient access to specialty care services. Our results controlling for general anxiety suggest that associations with worry about affording care are not solely related to general anxiety. Moreover, general anxiety might have the opposite relationship with healthcare services use, i.e., increase specialty care utilization. Our findings are 
Table 2 Associations Between Material Financial Burden, Worry About Affording Care, General Anxiety, and Healthcare Utilization

\begin{tabular}{llll}
\hline \hline Predictors & Outcome & Relative Risk & 95\% confidence interval \\
\hline Worry about affording healthcare services & ER visits & 0.89 & $0.48,1.67$ \\
Worry about affording prescription drugs & & 0.77 & $0.41,1.48$ \\
General anxiety & & 0.90 & $0.53,1.54$ \\
Material financial risk & Urgent care visits & 1.33 & $0.47,3.76$ \\
Worry about affording healthcare services & & 0.99 & $0.65,1.52$ \\
Worry about affording prescription drugs & & 1.36 & $0.87,2.12$ \\
General anxiety & Primary care visits & 1.07 & $0.64,1.79$ \\
Material financial risk & & 0.46 & $0.19,1.12$ \\
Worry about affording healthcare services & & 0.84 & $0.63,1.12$ \\
Worry about affording prescription drugs & Specialty care visits & 0.77 & $0.56,1.05$ \\
General anxiety & & 1.02 & $0.69,1.51$ \\
Material financial risk & & 0.73 & $0.48,1.09$ \\
Worry about affording healthcare services & 0.46 & $0.28,0.75$ \\
Worry about affording prescription drugs & 0.62 & $0.38,1.01$ \\
General anxiety & 1.82 & $1.14,2.90$ \\
Material financial risk & 1.18 & $0.64,2.19$ \\
\hline
\end{tabular}

Each predictor was compared separately to each outcome. Analyses adjusted for the value of the outcome for the 12 months prior, age, gender, racelethnicity, Neighborhood Deprivation Index, language, and cost-sharing subsidy. Italics indicates significance at the $p<0.05$ level

consistent with other research examining measures of anxiety and worry suggesting measures need to be specific. ${ }^{18-20}$ Overall, these results suggest that screening for worry about affording healthcare may be a helpful addition to other social risk screening.

We found that worry about affording prescriptions was not related to any measure of healthcare utilization included in the analysis. This might be because we only measured utilization, not prescription use, in this study. Worry about affording prescriptions might instead be associated with healthcare behaviors, such as medication rationing or pill-splitting. Medication adherence was not measured in this study because participants had a variety of medical conditions and adherence could not be standardized. A broader array of health-related outcomes should be included in future research on this topic.

This study had several limitations. First, we were unable to include a covariate for medical diagnoses because around $82 \%$ of the sample were missing data for the comorbidity index. We were also unable to determine whether healthcare utilization was consistent with recommended guidelines (e.g., preventive screenings) or not. Due to limitations in the survey length, we were not able to use the fully validated Worry about Affording Healthcare Scale. Because of the nature of the data, results do not necessarily imply that worry about affording healthcare is causally related to healthcare utilization. The sample size and response rate were relatively small. We also did not examine the impact of worry about affording healthcare on inpatient hospitalizations as hospitalizations were relatively rare in this population. We did not investigate the association between healthcare utilization and subsequent financial worry; prior research studies have shown that healthcare utilization could lead to worry about affording healthcare. ${ }^{10,36}$ However, we did include bivariate associations of baseline healthcare use with the financial and anxiety survey variables in the Appendix. Lastly, the sample consisted of healthcare exchange members in California so results may not generalize to patients

Table 3 Associations Between Worry About Affording Healthcare and Healthcare Use, Controlling for Material Financial Risk, and General Anxiety.

\begin{tabular}{llll}
\hline \hline Predictor & Outcome & Relative risk & 95\% confidence interval \\
\hline Worry about affording healthcare services & ER visits & 1.00 & $0.50,2.01$ \\
Worry about affording prescription drugs & & 0.64 & $0.32,1.29$ \\
General anxiety & & 0.82 & $0.44,1.52$ \\
Material financial risk & Urgent care visits & 1.79 & $0.66,4.84$ \\
Worry about affording healthcare services & & 0.72 & $0.41,1.28$ \\
Worry about affording prescription drugs & & 2.01 & $1.14,3.55$ \\
General anxiety & Primary care visits & 0.38 & $0.15,0.95$ \\
Material financial risk & & 1.28 & $0.78,2.12$ \\
Worry about affording healthcare services & & 1.00 & $0.69,1.46$ \\
Worry about affording prescription drugs & Specialty care visits & 0.78 & $0.51,1.18$ \\
General anxiety & & 1.14 & $0.77,1.69$ \\
Material financial risk & & 0.79 & $0.51,1.24$ \\
Worry about affording healthcare services & 0.40 & $0.25,0.64$ \\
Worry about affording prescription drugs & 0.98 & $0.67,1.45$ \\
General anxiety & 1.98 & $1.23,3.18$ \\
Material financial risk & 1.26 & $0.68,2.32$ \\
\hline
\end{tabular}

All predictors were entered into the same regression models. Analyses adjusted for the value of the outcome for the 12 months prior, age, gender, racelethnicity, Neighborhood Deprivation Index, language, and cost-sharing subsidy. Italics indicates significance at the $p<0.05$ level 
receiving insurance coverage from other sources or geographic areas.

Further research on worry about affording healthcare services can help clarify distinctions between these interrelated concepts. Financial interventions such as financial counseling, ${ }^{37}$ financial navigation, ${ }^{38}$ and costs of care conversations ${ }^{39}$ have been developed but need to be studied to determine their impacts on both material and psychological financial risks. A program in Toronto screened primary care patients for financial burden and provided a list of specific resources based on patient responses. ${ }^{40}$ As worry is often driven by uncertainty, ${ }^{24}$ interventions that connect patients to resources or provide cost information early in the care trajectory may reduce uncertainty about future costs. Research will also need to better explore the feasibility of delivering these types of interventions in healthcare settings.

\section{CONCLUSIONS}

Overall, our results suggest associations between worry about affording healthcare services and specialty healthcare utilization. Cost of care concerns were not associated with primary care utilization. These findings were independent of both actual material financial risk and general anxiety. General anxiety was associated with increased specialty care utilization. Future research is needed to determine the best way to intervene on psychological vs. material financial risk and how interventions can be targeted to both influence healthcare utilization and improve patient outcomes.

Supplementary Information The online version contains supplementary material available at https://doi.org/10.1007/s11606-02006479-6.

Acknowledgments: The authors would like to thank the staff of Kaiser Permanente Washington, Southern California, and Colorado for contributing to this project.

Corresponding Author: Salene M. W. Jones, PhD, MA; Fred Hutchinson Cancer Research Center, Seattle, WA, USA (e-mail: smjones3@fredhutch.org).

Funding Funding was provided by the Kaiser Foundation.

\section{Compliance with ethical standards:}

Conflict of Interest: The authors declare that they do not have a conflict of interest.

\section{REFERENCES}

1. Irwin $\mathbf{A}$, Valentine $\mathbf{N}$, Brown $\mathbf{C}$, et al. The commission on social determinants of health: Tackling the social roots of health inequities. Plos Medicine. 2006;3(6):749-751.

2. Heiman HJ, Artiga S. Beyond health care: The role of social determinants in promoting health and health equity. Kaiser Family Foundation;2015.

3. Goldstein D, Holmes J. 2011 Physicians' Daily Life Report. Robert Wood Johnson Foundation;2011.
4. Kennedy BP, Kawachi I, Glass R, Prothrow-Stith D. Income distribution, socioeconomic status, and self rated health in the United States: multilevel analysis. BMJ. 1998;317(7163):917-921.

5. Xiao Q, Berrigan D, Matthews CE. A prospective investigation of neighborhood socioeconomic deprivation and self-rated health in a large US cohort. Health Place. 2017;44:70-76.

6. Ota A, Yatsuya H, Nishi N, et al. Relationships among Socioeconomic Factors and Self-rated Health in Japanese Adults: NIPPON DATA2010. $J$ Epidemiol. 2018;28 Suppl 3:S66-S72.

7. Sulander T, Pohjolainen P, Karvinen E. Self-rated health (SRH) and socioeconomic position (SEP) among urban home-dwelling older adults. Arch Gerontol Geriatr. 2012;54(1):117-120.

8. Koch CG, Li L, Kaplan GA, et al. Socioeconomic Position, Not Race, Is Linked to Death After Cardiac Surgery. Circulation: Cardiovascular Quality and Outcomes. 2010;3(3):267-276.

9. Valtorta NK, Hanratty B. Socioeconomic variation in the financial consequences of ill health for older people with chronic diseases: a systematic review. Maturitas. 2013;74(4):313-333.

10. Yabroff KR, Dowling EC, Guy GP, et al. Financial Hardship Associated With Cancer in the United States: Findings From a Population-Based Sample of Adult Cancer Survivors. J Clin Oncol. 2016;34(3):259-267.

11. Altice CK, Banegas MP, Tucker-Seeley RD, Yabroff KR. Financial Hardships Experienced by Cancer Survivors: A Systematic Review. J Natl Cancer Inst. 2017; 109(2).

12. Gordon LG, Merollini KMD, Lowe A, Chan RJ. A Systematic Review of Financial Toxicity Among Cancer Survivors: We Can't Pay the Co-Pay. Patient. 2017; 10(3):295-309.

13. Bestvina CM, Zullig LL, Rushing $\mathbf{C}$, et al. Patient-oncologist cost communication, financial distress, and medication adherence. $J$ Oncol Pract. 2014; 10(3): 162-167.

14. Zullig LL, Peppercorn JM, Schrag D, et al. Financial Distress, Use of Cost-Coping Strategies, and Adherence to Prescription Medication Among Patients With Cancer. J Oncol Pract. 2013;9(6S):60s-63s.

15. Khera N, Chang YH, Hashmi S, et al. Financial burden in recipients of allogeneic hematopoietic cell transplantation. Biol Blood Marrow Transplant. 2014;20(9): 1375-1381.

16. Jones S, Du YX, Panattoni L, Henrikson N. Development of a questionnaire to assess worry about affording healthcare in an international sample. Qual Life Res. 2019;28:S54-S54.

17. Jones SMW, Walker R, Fujii M, Nekhlyudov L, Rabin BA, Chubak J. Financial difficulty, worry about affording care, and benefit finding in long-term survivors of cancer. Psychooncology. 2018;27(4):1320-1326.

18. Berggren U. General and specific fears in referred and self-referred adult patients with extreme dental anxiety. Behav Res Ther. 1992;30(4):395401.

19. Buss C, Davis EP, Hobel CJ, Sandman CA. Maternal pregnancy-specific anxiety is associated with child executive function at 6-9 years age. Stress. 2011;14(6):665-676.

20. Reck C, Zimmer K, Dubber S, Zipser B, Schlehe B, Gawlik S. The influence of general anxiety and childbirth-specific anxiety on birth outcome. Arch Womens Ment Health. 2013;16(5):363-369.

21. Eastin MS, Guinsler NM. Worried and wired: effects of health anxiety on information-seeking and health care utilization behaviors. Cyberpsychol Behav. 2006;9(4):494-498.

22. Norris L. Will you receive an Obamacare premium subsidy? How the Affordable Care Act's subsidies are calculated, and who is eligible to receive them. HealthInsurance.org. https://www.healthinsurance.org/ obamacare/will-you-receive-an-obamacare-premium-subsidy/. Published 2018. Accessed June 1, 2019.

23. Collins SR, Bhupal HK, Doty MM. Health insurance coverage eight years after the ACA. The Commonwealth Fund. https://www. commonwealthfund.org/publications/issue-briefs/2019/feb/health-insurance-coverage-eight-years-after-aca. Published 2019. Accessed.

24. Barlow DH. Anxiety and its disorders: The nature and treatment of anxiety and panic. 2nd ed. New York: The Guilford Press; 2004.

25. Jones SM, Amtmann D. Differential item function analysis of a scale measuring worry about affording healthcare in multiple sclerosis. Rehabil Psychol. 2016;61(4):430-434.

26. Jones SM, Amtmann D. Health care worry is associated with worse outcomes in multiple sclerosis. Rehabil Psychol. 2014;59(3):354-359.

27. LaForge $\mathbf{K}$, Gold R, Cottrell E, et al. How 6 Organizations Developed Tools and Processes for Social Determinants of Health Screening in Primary Care: An Overview. The Journal of ambulatory care management. 2018;41(1):2-14.

28. Adler NE, Stead WW. Patients in context-EHR capture of social and behavioral determinants of health. $N$ Engl J Med. 2015;372(8):698-701. 
29. Puterman E, Haritatos J, Adler NE, Sidney S, Schwartz JE, Epel ES Indirect effect of financial strain on daily cortisol output through daily negative to positive affect index in the Coronary Artery Risk Development in Young Adults Study. Psychoneuroendocrinology. 2013;38(12):28832889.

30. Hall MH, Matthews KA, Kravitz HM, et al. Race and financial strain are independent correlates of sleep in midlife women: the SWAN sleep study. Sleep. 2009;32(1):73-82.

31. Lowe B, Wahl I, Rose M, et al. A 4-item measure of depression and anxiety: validation and standardization of the Patient Health Questionnaire-4 (PHQ-4) in the general population. J Affect Disord. 2010; 122(1-2):86-95.

32. Zeger SL, Liang KY, Albert PS. Models for longitudinal data: a generalized estimating equation approach. Biometrics. 1988;44(4): 10491060.

33. Lesaffre E, Marx BD. Collinearity in Generalized Linear-Regression. Commun Stat-Theor M. 1993;22(7):1933-1952.

34. Lewis CC, Wellman R, Jones SMW, et al. Comparing the performance of two social risk screening tools in a vulnerable subpopulation. Journal of Family Medicine and Primary Care. In press.
35. Horst P. The role of predictor variables which are independent of the criterion. Social Science Research Council. 1941;48:431-436.

36. Jones SMW, Litwin P. Association of healthcare utilization with financial worry and anxiety in a general population sample. Psychol Health Med. 2020:1-8.

37. Collins JM, Olive P, O'Rourke CM. Financial Coaching's Potential for Enhancing Family Financial Security. Journal of Extension. 2013;51(1).

38. Shankaran V, Leahy T, Steelquist J, et al. Pilot Feasibility Study of an Oncology Financial Navigation Program. J Oncol Pract. 2018;14(2):e122e129.

39. Henrikson NB, Tuzzio L, Loggers ET, Miyoshi J, Buist DS. Patient and oncologist discussions about cancer care costs. Support Care Cancer. 2014:22(4):961-967.

40. Pinto AD, Bondy M, Rucchetto A, Ihnat J, Kaufman A. Screening for poverty and intervening in a primary care setting: an acceptability and feasibility study. Fam Pract. 2019;36(5):634-638

Publisher's Note: Springer Nature remains neutral with regard to jurisdictional claims in published maps and institutional affiliations. 